Published in final edited form as:

J Am Chem Soc. 2016 October 12; 138(40): 13167-13170. doi:10.1021/jacs.6b08512.

\title{
Enzymatic Reductive Dehalogenation Controls the Biosynthesis of Marine Bacterial Pyrroles
}

\author{
Abrahim El Gamal ${ }^{\dagger}$, Vinayak Agarwal ${ }^{\dagger}$, Imran Rahman $^{\dagger}$, and Bradley S. Moore ${ }^{\star}, \dagger, \ddagger$ \\ ${ }^{\dagger}$ Center for Oceans and Human Health, Scripps Institution of Oceanography, Scripps Institution of \\ Oceanography, University of California, San Diego, San Diego, California 92093, United States \\ ¥Skaggs School of Pharmacy and Pharmaceutical Sciences, University of California, San Diego, \\ San Diego, California 92093, United States
}

\begin{abstract}
Enzymes capable of performing dehalogenating reactions have attracted tremendous contemporary attention due to their potential application in the bioremediation of anthropogenic polyhalogenated persistent organic pollutants. Nature, in particular the marine environment, is also a prolific source of polyhalogenated organic natural products. The study of the biosynthesis of these natural products has furnished a diverse array of halogenation biocatalysts, but thus far no examples of dehalogenating enzymes have been reported from a secondary metabolic pathway. Here we show that the penultimate step in the biosynthesis of the highly brominated marine bacterial product pentabromopseudilin is catalyzed by an unusual debrominase Bmp8 that utilizes a redox thiol mechanism to remove the $\mathrm{C}-2$ bromine atom of 2,3,4,5-tetrabromopyrrole to facilitate oxidative coupling to 2,4-dibromophenol. To the best of our knowledge, Bmp8 is first example of a dehalogenating enzyme from the established genetic and biochemical context of a natural product biosynthetic pathway.
\end{abstract}

Efforts toward the bioremediation of man-made polyhalogenated persistent organic pollutants have led to the identification of microbes and associated enzymes capable of dehalogenation of a wide range of organohalogens.(1-3) Nature is also a prolific source of polyhalogenated natural products ranging from simple aliphatic and aromatic molecules to complex terpenoids, oligopeptides, alkaloids, and polyketides with diverse biological properties.(4) Although numerous natural product halogenases have been characterized, here we report, to the best of our knowledge, the first example of a dedicated dehalogenating enzyme participating in the construction of a natural product.

The terminal reaction in the biosynthesis of the highly brominated marine bacterial natural product, pentabromopseudilin (1) by the marine bacterial Bmp pathway involves the biradical C-C coupling of 2,3,4-tribromopyrrole (2) to 2,4-dibromophenol catalyzed by a

*Corresponding author: bsmoore@ ucsd.edu.

Supporting Information

The Supporting Information contains experimental details, Table S1, Figures S1-S9, and details on compound characterization.

Statement of Competing Interests

The authors declare no competing financial interests. 
cytochrome P450(CYP450) Bmp7 (Figure 1A,B).(5) Although we previously confirmed the biosynthesis of 2,4-dibromophenol by enzymes Bmp5-6, the steps leading to the biosynthesis of 2 remained uncertain (Figure 1B). We demonstrated that Bmp1-4 synthesizes the symmetrical natural product 2,3,4,5-tetrabromopyrrole (3), which is not a substrate for Bmp7, implying that an as yet uncharacterized dehalogenation event must occur to furnish 2 from 3 en route the assembly of 1 (Figure 1B).(5, 6) All Bmp biosynthetic enzymes have been characterized with the exception of an orphan $\sim 21 \mathrm{kDa}$ protein with distant homology to thioredoxin/alkyl hydroperoxidase AhpD encoded by the open reading frame (ORF) bmp8, located upstream of CYP450 electron transport partner-encoding bmp910 (Figure 1A).(5, 6) Notably, bmp8 homologues are absent in the bmp gene cluster recently identified from Pseudoalteromonas sp. PS5 that produces the coral larval settlement cue 3, and not 2, as its dominant polybrominated pyrrole metabolite (Figure 1A).(6) Thus, we reasoned that Bmp8 might support the reductive dehalogenation of 3 to 2 . To test this hypothesis in vitro, we first sought to purify Bmp8 as a soluble recombinant protein.

Initial attempts to prepare recombinant Bmp8 were frustrated by the insolubility of recombinantly expressed protein. Working on the assumption that the product of the Bmp1 didomain protein is the substrate of Bmp8 and thus that these two proteins may form a functional complex, we coexpressed the N-His6-tagged-Bmp8 from M. mediterranea MMB-1 with the untagged Bmp1 thioesterase (TE) domain. Purification by affinity chromatography led to the isolation of Bmp8 as a stand-alone, soluble protein (Figure S1). With purified Bmp8 protein in hand, we next sought to test its activity against 3 .

We first inspected the domain architecture of Bmp8 to ascertain the assay components necessary to reconstitute its activity. Consistent with its homology to the mycobacterial thioredoxin-like enzyme AhpD, the primary amino acid sequence of Bmp8 contains a conserved thioredoxin Cys-X-X-Cys motif (CXXC; Cys82-X-X-Cys85; Figure 2A).(7) In the reaction mechanism for thioredoxins, the CXXC cysteinyl thiols deliver two electrons to the oxidant substrate leading to the formation of an intramolecular disulfide bond (Figure 2B). $(7,8)$ Hence, we reasoned that, so long as the Bmp8 CXXC was available in its reduced form, conversion of 3 to 2 should proceed without the requirement for exogenous redox cofactors. Indeed, incubation of Bmp8 with a 2-fold molar excess of 3 led to partial conversion to the product 2 confirmed by comparison to an authentic synthetic standard, while no conversion was observed for 3 incubated in the absence of enzyme over the same reaction period (Figure $2 \mathrm{C}$ curves "i" and "iii", and Figure S2). We next reasoned that if a disulfide bridge were formed at the Bmp8 active site CXXC following the reduction of 3 to 2 , then the enzyme activity should be restored by the addition of a thiol reducing agent leading to complete conversion of the substrate 3 in 2-fold molar excess of the enzyme to the product 2. Indeed, inclusion of the thiol reductant glutathione ("GSH" in figures) in excess to enzyme and product led to complete conversion of 3 to 2 , whereas only slight conversion of 3 to 2 was observed for the no-enzyme negative control when glutathione was present in the buffer (Figure $2 \mathrm{C}$ curves "ii" and "iv"). We further showed that Bmp8 is active under anaerobic conditions (Figure S3). Thus, we demonstrated the physiological route for the biosynthesis of the natural product 2 via enzymatic dehalogenation of 3 catalyzed by Bmp8. 
In addition to 2, a minor product with molecular formula $\mathrm{C} 4 \mathrm{NH} 3 \mathrm{Br} 2$ was observed and confirmed to be the symmetrical 3,4-dibromopyrrole (4) by 1H NMR (Figure 2C curve "iv" and Figure S2). Because of the formation of 4, we further investigated if 2 might also be a substrate for Bmp8. Indeed, incubation of Bmp8 with 2 along with excess glutathione led to only a partial conversion to 4 over the same incubation time for which 3 was completely converted to 2 and 4, whereas no conversion of 2 to 4 was observed in the corresponding noenzyme negative control (Figure 2C curves "v" and "vi"). Taken together, these results demonstrate that Bmp8 regioselectively dehalogenates the symmetrical 2/5-position of 3 to afford Bmp7 substrate 2. The activity of Bmp8 reconciles an earlier isotope feeding study that demonstrated a requirement of a symmetrical intermediate of unknown structure derived from 1-proline (i.e., 3) in the biosynthesis of 1.(9)

We next investigated the role of the CXXC motif in the catalytic cycle of Bmp8 by monitoring of its redox state by LC-MS. Following incubation with 3 in the absence of a reducing agent, we treated the intact Bmp8 with the thiol alkylating agent iodoacetamide (IAA) that specifically labels reduced cysteinyl thiols, thus protecting them from oxidation by molecular oxygen. Subsequent to alkylation, the protein was digested with trypsin and analyzed by LC-MS (Figure 3A(i)). The Bmp8 CXXC residues are present on a single predicted trypsin peptide fragment containing no additional cysteines (Figure 2A). Hence, dialkylation of the predicted CXXC-containing peptide fragment indicates the cysteine side chain thiols are preserved in their reduced state as shown in Figure $3 \mathrm{~A}(\mathrm{i})(-2 \mathrm{H}+2 \mathrm{AA})$. Meanwhile, the fragment less two protons $(-2 \mathrm{H})$ is indicative of oxidation giving rise to an intramolecular disulfide bridge (Figure 3A(i)). For Bmp8 incubated with 3, following trypsin digestion, we detected a peptide with mass corresponding to the $-2 \mathrm{H} \mathrm{Bmp8} \mathrm{CXXC}$ fragment, while no mass corresponding to the dialkylated Bmp8 CXXC peptide fragment could be observed (Figure 3A(ii) and Figure S4). In contrast, for the reaction incubated in the absence of 3 , we detected only the dialkylated peptide mass and not the $-2 \mathrm{H}$ peptide mass (Figure 3A(ii) and Figure S4). Taken together, these results are consistent with formation of an intramolecular disulfide bridge between Cys82 and Cys85 resulting from treatment with 3. Hence, we conclude that the two electrons required for the reduction of 3 to 2 are likely derived from the Bmp8 CXXC. We further examined the fates of two additional cysteine residues conserved among Bmp8 homologues from the contexts Bmp pathways found in public genomic databases, and confirmed that their oxidation states in Bmp8 were not affected by treatment with 3 (Figure S5).

Having demonstrated the suicide oxidation of Bmp8 coincident with the reductive dehalogenation of 3 to 2, we proceeded to interrogate the catalytic functions of the two cysteine residues in the Bmp8 CXXC motif residues by assaying the individual Cys to Ala mutants (i.e., Bmp8:C82A and Bmp8:C85A). Incubation of Bmp8:C82A with a 2-fold molar excess of 3 and glutathione led to complete conversion to products 2 and 4 identical to the wild-type enzyme activity (Figure 3B curves "i" and "ii"). Only a slight conversion to 2 was observed for Bmp8:C85A incubated with 3 that was not significantly different from the noenzyme negative control (Figure 3B curves "iii" and "iv"). In the absence of glutathione, we also observed conversion of 3 to 2 by Bmp8:C82A at a level comparable to the wild-type enzyme (Figure 3B curves "v" and "vi"). Taken together, our findings demonstrate that 
Cys85 is catalytically essential, whereas Cys82 appears to be nonessential to catalysis. Functionally, these results imply that Cys85 is the electron donor of the CXXC pair.

We next sought to identify the electron acceptor of the substrate 3. Assuming a 2-electron transfer, the recipient of the Cys85 electrons would either be the C-2-bromine substituent or the $\mathrm{C}-2$ position of 3 , leading, respectively, to the formation of a bromide or pyrrole adduct with the Cys85 side chain sulfur. In the absence of a resolving thiolate, turnover of 3 to 2 would only be observed if the electron acceptor were the $\mathrm{C}$-2-bromine, as the alternative route would lead to sequestration of the pyrrole by the Bmp8 and result in no turnover. Hence, the fact that Bmp8:C82A exhibits turnover in the absence of glutathione favors formation of a transient Cys 85 bromide thioether (Figure 3B(vi)). To investigate this mechanistic hypothesis, we monitored the mass of Bmp8:C82A incubated with and without a 2 -fold excess of 3 in the absence of glutathione by mass spectrometry. Following incubation with 3 , we observed a mass shift of +32 Da consistent with formation of a cysteinyl sulfinic acid (Cys-S-O2H), whereas no mass shift was observed for Bmp8:C82A incubated in the absence of 3 over the same time period (Figure 3C(i), Figure S6, and Table S1). Additionally, no mass shift was observed for inactive Bmp8:C85A treated in an identical manner, implying that change in mass observed in Bmp:C82A corresponds to a modification of Cys85 (Figure 3C(ii), Figure S6, and Table S1). Free cysteinyl halide rapidly hydrolyzes to sulfenic acid (Cys-S-OH), which in turn quickly oxidizes to sulfinic acid under nonreducing aerobic conditions.(10) Hence, the formation of a Cys85 sulfinic acid is consistent with formation of a Cys85 halide species.

To deconvolute further the oxidation of the Cys 85 side chain sulfur, we incubated Bmp8:C82A in buffer containing an excess of alkylating agent dimedone that selectively labels cysteinyl sulfenic acid to form a stable cysteinyl dimedone thioether.(11) Incubation with 3 led to a mass shift of +138 Da consistent with a dimedone adduct, along with minor population corresponding to the sulfinic acid species anticipated due to competition with molecular oxygen, whereas no mass shift was observed for Bmp8:C82A incubated without 3 (Figure 3C(iii), Figure S6, and Table S1). Notably, inclusion of dimedone in the reaction buffer did not discernibly affect the activity of Bmp8:C82A indicating that higher oxidation states than sulfenic acid do not arise from further turnover of 3 (Figure S7). Taken together, these results imply formation of a Cys 85 bromide thioether by proxy of an observable Cy85 sulfenic acid, corroborating the $\mathrm{C}$-2-bromine substituent of 3 as the electron acceptor.

On the basis of our biochemical experiments, we propose an unanticipated mechanism for the reductive dehalogenation of bromopyrroles by Bmp8 (Scheme 1). Protonation of C-2 of 3 would first lead to formation of a pyrrole iminium diene intermediate in which the C-2bromine would be eliminated by an E1-type attack of the cysteinyl thiolate of Cys85 leading to the formation of 2 and cysteinyl bromide. The resulting Cys 85 bromide is then resolved to bromide and an intramolecular Cys82-Cys85 disulfide bond by one of two routes, either direct thiolysis or sequential hydrolysis followed by thiolysis of the thioether bond (paths " $i$ " and "ii", respectively, in Scheme 1). Although no dimedone adduct was observed for wildtype Bmp8 incubated with 3 (Figure S6 and Table S1), as cysteinyl thiolates react rapidly with cysteinyl sulfenic acids to form disulfide bonds, either route may be operative. (10) 
The proposed mechanism for Bmp8 is analogous to enzymatic dehalogenation mechanisms invoking direct attack of a genetically encoded or prosthetic group electron donors on a halonium leaving group. For example, the peroxiredoxin-like mechanism favored for thyroid hormone aromatic deiodinase involves the formation of a selenyl iodide with a selenocysteine residue side chain selenolate that is proposed to resolve to a selenyl sulfide by an endogenous cysteine residue.(12) Formation of an active site cysteinyl bromide has also been proposed for the dehalogenation of the nonphysiological substrate 5-bromouridine by thymidylate synthase.(13) Lastly, formation of cob(II)alamin halide adduct was recently suggested from structure-function studies of two bacterial reductive dehalogenases.

The characterization of Bmp8 demonstrates the first example of a dedicated enzymatic dehalogenation reaction in a natural product biosynthetic pathway. The debromination of 3 unmasks the reactive $\mathrm{C}-2$ position in 2 for subsequent Bmp7-catalyzed oxidative coupling to form hexabromobipyrrole as well as 1.(5) This explicit enzymatic dehalogenation contrasts with past examples of dehalogenation chemistry in natural products biosynthesis following from cryptic substrate-activating halogenation reactions.(15-18) Further, the cofactorindependent mechanism of Bmp8 is unique among bacterial dehalogenases characterized to date that require cofactors such as cobalamin, FMN, and glutathione.(12) Of note, while glutathione occurs at high $(\mathrm{mM})$ intracellular concentrations, other surrogate electron donors to Bmp8 may also be operative in vivo.(19) For example, peroxidase activity of AhpD can be recycled by the flavoprotein reductase $\mathrm{AhpF}$ for which homologues are present in Bmpharboring strains.(20) Moreover, it is possible that off-pathway small protein thiols might contribute to in vivo conversion of 3 to 2, though we did not observe dehalogenation of 3 upon incubation with the unrelated thioredoxin TrxA from Escherichia coli (Figure S8).

A query of publically available sequence databases for Bmp8 homologues identified numerous homologues of unknown function from diverse bacterial genera and genetic contexts (Figure S9). Although the majority of dehalogenases to date have been identified on the basis of their ability to dehalogenate anthropogenic organohalogens, Bmp8 presents a new class of dehalogenase from the uncharted frontier of natural products biosynthesis.

\section{Supplementary Material}

Refer to Web version on PubMed Central for supplementary material.

\section{Acknowledgments}

We thank our colleagues Stefan Diethelm and Zachary Miles for advice and critical reading of the paper. This work was jointly supported by the US National Science Foundation (OCE-1313747) and the US National Institute of Environmental Health Sciences (P01-ES021921) through the Ocean and Human Health Program to B.S.M., the US National Institutes of Health Marine Biotechnology Training Grant pre-doctoral fellowship to A.E. (T32GM067550), the Helen Hay Whitney Foundation postdoctoral fellowship and US National Institute of Environmental Health Sciences (K99-ES026620) to V.A..

\section{References}

1. Smidt H, de Vos WM. Annu Rev Microbiol. 2004; 58:43.doi: 10.1146/annurev.micro. 58.030603.123600 [PubMed: 15487929] 
2. Janssen DB, Oppentocht JE, Poelarends G. J Curr Opin Biotechnol. 2001; 12:254.doi: 10.1016/ S0958-1669(00)00208-1

3. Copley SD. Nat Chem Biol. 2009; 5:559.doi: 10.1038/nchembio.197 [PubMed: 19620997]

4. Gribble, GW. Naturally Occurring Organohalogen Compounds - A Comprehensive Update. Vol. 91. Springer; Vienna: 2010.

5. Agarwal V, El Gamal AA, Yamanaka K, Poth D, Kersten RD, Schorn M, Allen EE, Moore BS. Nat Chem Biol. 2014; 10:640.doi: 10.1038/nchembio.1564 [PubMed: 24974229]

6. El Gamal A, Agarwal V, Diethelm S, Rahman I, Schorn MA, Sneed JM, Louie GV, Whalen KE, Mincer TJ, Noel JP, Paul VJ, Moore BS. Proc Natl Acad Sci U S A. 2016; 113:3797.doi: 10.1073/ pnas.1519695113 [PubMed: 27001835]

7. Nunn CM, Djordjevic S, Hillas PJ, Nishida CR, Ortiz de Montellano PR. J Biol Chem. 2002; 277:20033.doi: 10.1074/jbc.M200864200 [PubMed: 11914371]

8. Fomenko DE, Gladyshev VN. Biochemistry. 2003; 42:11214.doi: 10.1021/bi034459s [PubMed: 14503871]

9. Peschke JD, Hanefeld U, Laatsch H. Biosci, Biotechnol, Biochem. 2005; 69:628.doi: 10.1271/bbb. 69.628 [PubMed: 15784994]

10. Nagy P, Ashby MT. J Am Chem Soc. 2007; 129:14082.doi: 10.1021/ja0737218 [PubMed: 17939659]

11. Allison WS. Acc Chem Res. 1976; 9:293.doi: 10.1021/ar50104a003

12. Schweizer U, Steegborn C. J Mol Endocrinol. 2015; 55:R37.doi: 10.1530/JME-15-0156 [PubMed: 26390881]

13. Carreras CW, Santi DV. Annu Rev Biochem. 1995; 64:721.doi: 10.1146/annurev.bi. 64.070195.003445 [PubMed: 7574499]

14. Jugder BE, Ertan H, Lee M, Manefield M, Marquis CP. Trends Biotechnol. 2015; 33:595.doi: 10.1016/j.tibtech.2015.07.004 [PubMed: 26409778]

15. Vaillancourt FH, Yeh E, Vosburg DA, O’Connor SE, Walsh CT. Nature. 2005; 436:1191.doi: 10.1038/nature03797 [PubMed: 16121186]

16. Yamanaka K, Ryan KS, Gulder TA, Hughes CC, Moore BS. J Am Chem Soc. 2012; 134:12434.doi: 10.1021/ja305670f [PubMed: 22800473]

17. Neumann CS, Walsh CT. J Am Chem Soc. 2008; 130:14022.doi: 10.1021/ja8064667 [PubMed: 18828590]

18. Gu L, Wang B, Kulkarni A, Geders TW, Grindberg RV, Gerwick L, Hakansson K, Wipf P, Smith JL, Gerwick WH, Sherman DH. Nature. 2009; 459:731.doi: 10.1038/nature07870 [PubMed: 19494914]

19. Masip L, Veeravalli K, Georgiou G. Antioxid Redox Signaling. 2006; 8:753.doi: 10.1089/ars. 2006.8.753

20. Hillas PJ, del Alba FS, Oyarzabal J, Wilks A, Ortiz De Montellano PR. J Biol Chem. 2000; 275:18801.doi: 10.1074/jbc.M001001200 [PubMed: 10766746] 
A

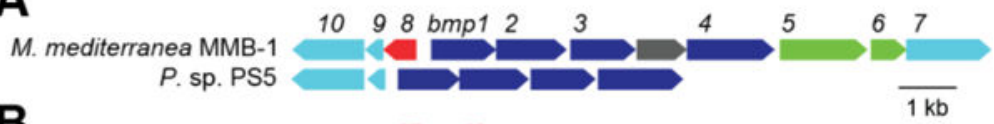

B

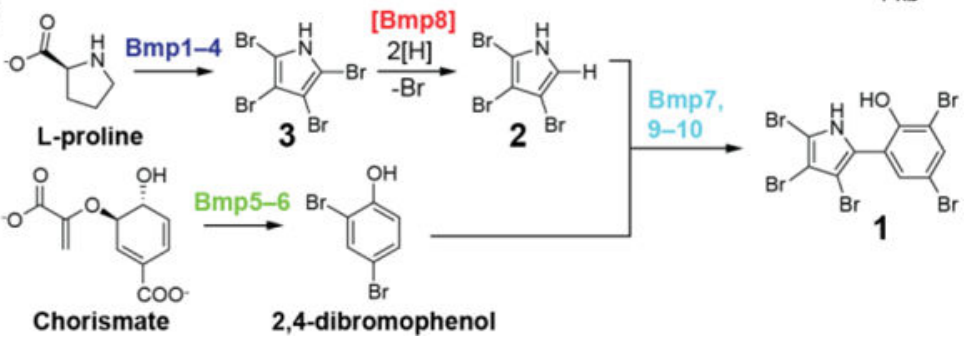

Figure 1.

(A) Bmp gene clusters from Marinomonas mediterranea MMB-1 and Pseudoalteromonas sp. PS5. The Bmp gene cluster from M. mediterranea MMB-1 features an ORF encoding a putative permease inserted between bmp3 and bmp4. (B) Biosynthesis of 1 with the hypothesized role of Bmp8. 


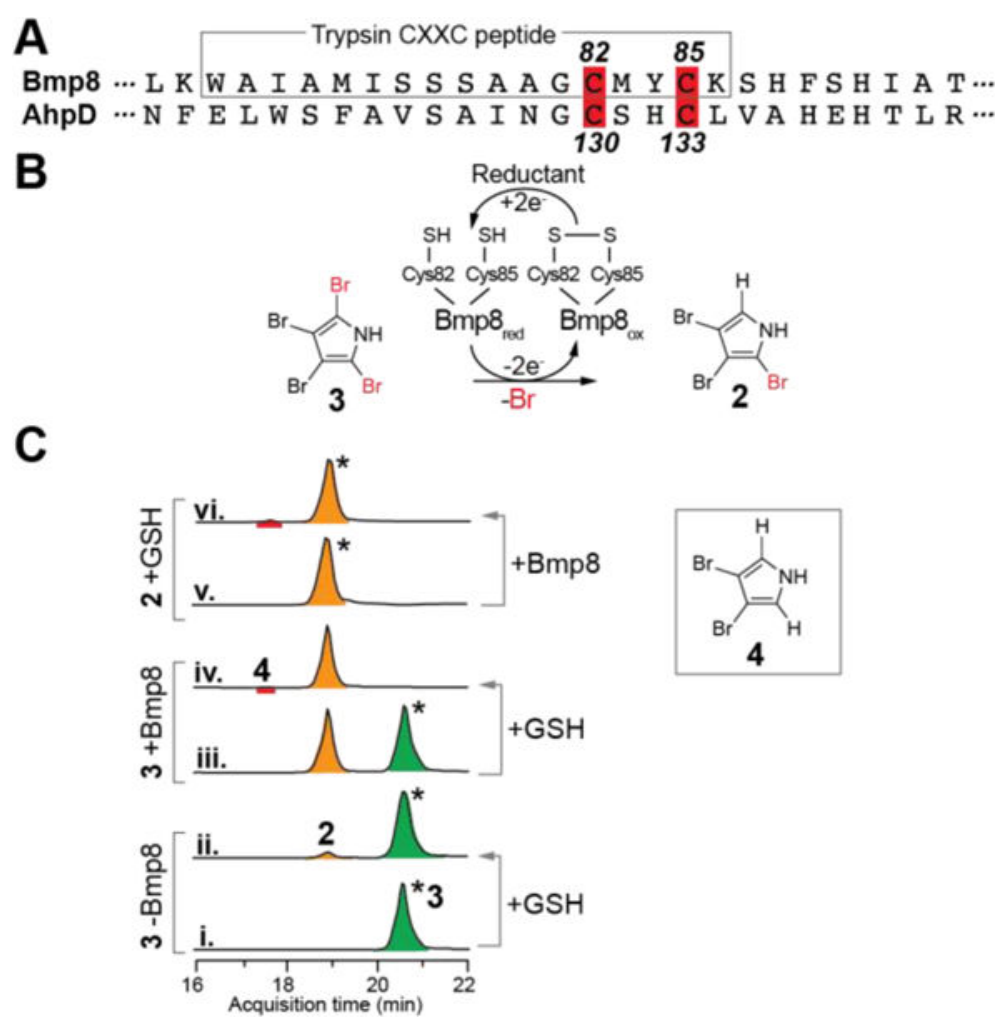

Figure 2.

(A) Portion of amino acid sequence alignment of M. mediterranea MMB-1 Bmp8 (GenBank WP_013663189.1) with Mycobacterium tuberculosis H37Rv AhpD (GenBank

AAA86657.1) highlighting conserved CXXC motif, and inclusive of the predicted CXXCcontaining trypsin peptide fragment for Bmp8; CXXC residues are numbered above for Bmp8 and below for AhpD. (B) Hypothesized thioredoxin-inspired redox mechanism for conversion of 3 to 2 by Bmp8. (C) Combined extracted ion chromatograms (EICs) for $\mathrm{m} / \mathrm{z}$ 's corresponding to $[\mathrm{M}-\mathrm{H}] 1-$ ions for 3 (green), 2 (orange), and 4 (red) for organic extracts of 1 $\mathrm{mL}$ reactions containing $100 \mu \mathrm{M} 3$ or 2 with (+) or without (-) $50 \mu \mathrm{M} \mathrm{Bmp8}$, and with (+) or without (-) $1 \mathrm{mM} \mathrm{GSH}$ in buffer ( $\mathrm{pH}$ 8) containing $20 \mathrm{mM}$ Tris- $\mathrm{HCl}, 50 \mathrm{mM} \mathrm{KCl}$, and $10 \%$ glycerol incubated at $25{ }^{\circ} \mathrm{C}$ for $30 \mathrm{~min}$.; synthetic starting material is indicated by an asterisk (*) above the respective peak. 


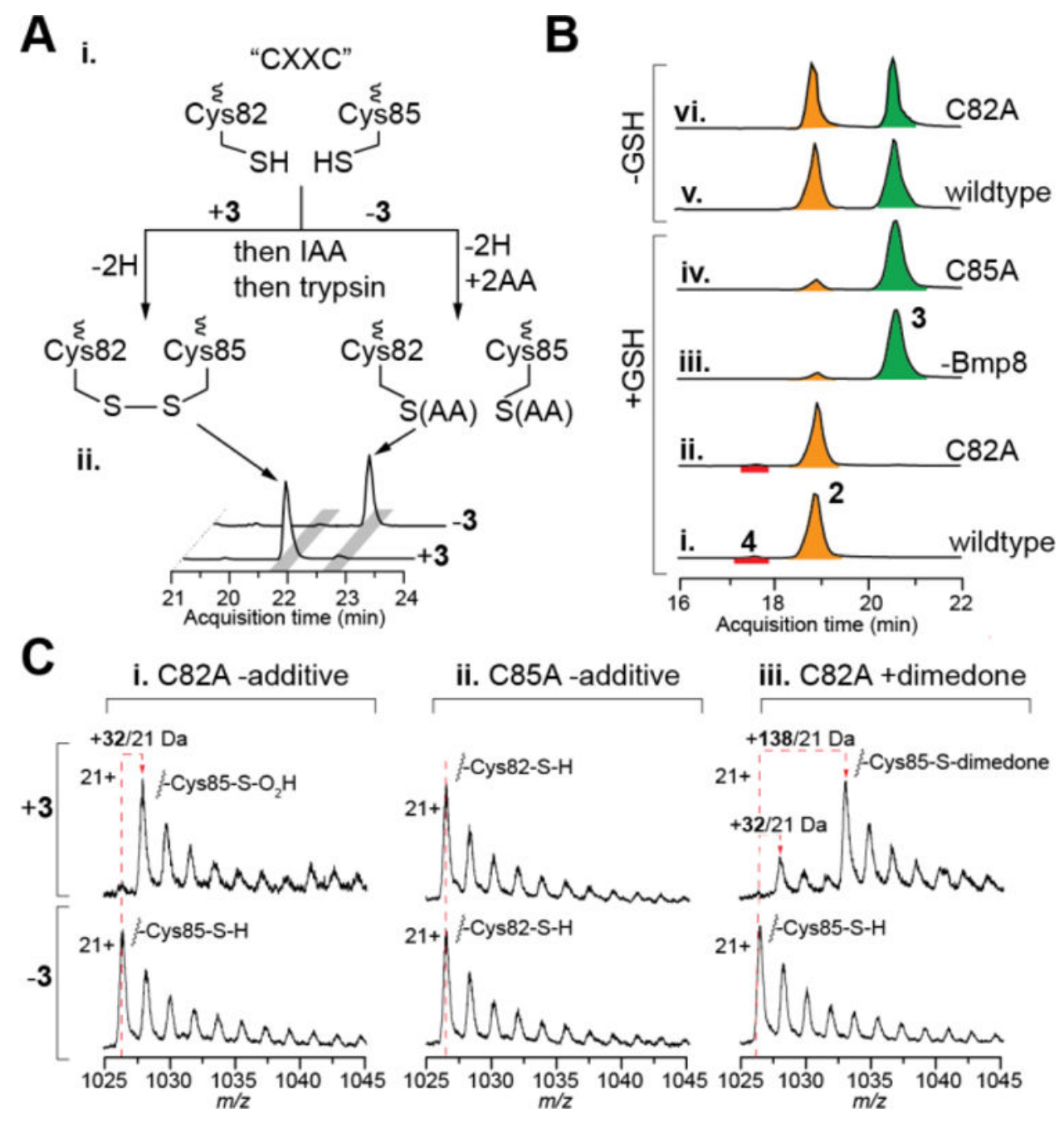

Figure 3.

(A) i. Experimental design for monitoring redox state of the Bmp8 CXXC, and ii. Combined EICs for $\mathrm{m} / \mathrm{z}$ 's for predicted $[\mathrm{M}+2 \mathrm{H}] 2+$ ions for the dialkylated $(+2 \mathrm{AA})$ and oxidized $(-2 \mathrm{H}) \mathrm{Bmp8} \mathrm{CXXC}$-containing trypsin peptide fragment shown in Figure 2A for Bmp8 incubated with (+) and without (-) 3. (B) Combined EICs for predicted m/z's of [M-H]1ions corresponding to 2 (orange), 3 (green), and 4 (red) for organic extracts of reactions with 3 as substrate conducted in an identical manner to that described in the caption of Figure $2 \mathrm{C}$. (C) Select mass spectra highlighting the $[\mathrm{M}+21 \mathrm{H}] 21+$ charge state for Bmp8 Cys to Ala mutants under treatments indicated in the panel. 

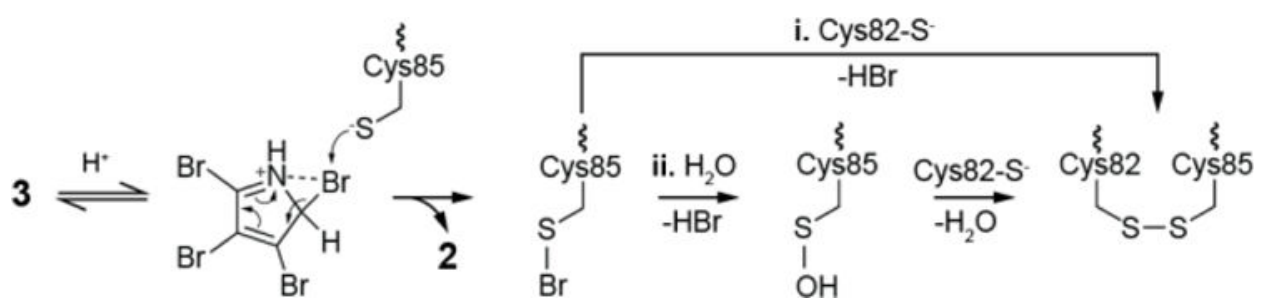

Scheme 1.

Bmp8 Mechanistic Proposal. 